\section{Database Administrator (DBA)}

M. Tamer Özsu

Cheriton School of Computer Science, University of Waterloo, Waterloo, ON, Canada

\section{Definition}

A database administrator (DBA) is a person with the overall responsibility to manage a database installation. The typical tasks performed by a DBA are database installation, configuration, performance monitoring, security and privacy management, backup and recovery, and database migration when needed. DBA uses a number of database configuration, tuning, and monitoring tools to achieve these tasks. It may be more appropriate to consider DBA as a "role" rather than a single person, as these tasks might be shared among multiple individuals.

\section{Historical Background}

Early database installations were reasonably small, and the DBMS software was relatively simple that end users could easily perform the tasks of a DBA. However, the size and complexity of these installations quickly grew as more applications were ported to database systems, while, at the same time, the DBMSs started providing rich feature sets. Managing this became a specialized job that now requires significant investment.

\section{Key Applications}

Database applications span almost all of the fields where large volumes of data are collected and managed. These installation require a DBA to run them.

\section{Cross-References}

Database Management System

$>$ Index Tuning

- Physical Layer Tuning

- Schema Tuning

\section{Recommended Reading}

1. Elmasri R, Navathe S. Fundamentals of database systems, 7th edn. Pearson; 2016.

2. Silberschatz A, Korth HF, Sudarshan S. Database system concepts, 6th edn. McGraw-Hill; 2010.

3. Ramakrishnan R, Gehrke J. Database management systems, 3rd edn. McGraw-Hill; 2002. 